\title{
Overexpressed HO-1 is associated with reduced STAT3 activation in preeclampsia placenta and inhibits STAT3 phosphorylation in placental JEG-3 cells under hypoxia
}

\author{
Hong-Mei Qu, Li-Ping Qu, Xiao-Yan Li, Xian-Zhen Pan
}

Department of Obstetrics and Gynecology, the Affiliated Yantai Yuhuangding Hospital of Qingdao University, Yantai, China

Submitted: 19 February 2016

Accepted: 8 August 2016

Arch Med Sci 2018; 14, 3: 597-607

DOI: https://doi.org/10.5114/aoms.2016.63261

Copyright $\odot 2016$ Termedia \& Banach

\section{Abstract}

Introduction: Inadequate trophoblast invasion and placentation are widely believed to contribute to preeclampsia, and multiple lines of evidence indicate the involvement of hypoxia in preeclampsia. However, the molecular mechanisms underlying the association of placental hypoxia with preeclampsia are not clear.

Material and methods: The present study focused on the role in preeclampsia of heme oxygenase 1 ( $\mathrm{HO}-1)$, which is an inducible isoform of $\mathrm{HO}$ in response to hypoxia, via examining the expression of $\mathrm{HO}-1$ and the expression and phosphorylation (Tyr705) of Signal transducer and activator of transcription (STAT) 3 in preeclamptic placentas via the immunohistochemical method, western blotting assay and RT-qPCR method. Then we investigated the regulation by HO- 1 of the expression and phosphorylation of STAT3 in human placental choriocarcinoma JEG-3 cells under hypoxia.

Results: There was upregulation of $\mathrm{HO}-1$ at both mRNA $(1.506 \pm 0.08347$ $(N=37)$ vs. $1.000 \pm 0.08854(N=31), p<0.0001)$ and protein $(0.630 \pm 0.155$ $(N=35)$ vs. $0.310 \pm 0.052,0.630 \pm 0.155(N=35), p<0.001)$ levels and a reduced level of STAT3 phosphorylation (Tyr 705) in the preeclamptic placental tissues, compared to normal placental tissues $(0.143 \pm 0.027(N=35)$ vs. $0.194 \pm 0.028(N=35), p<0.01)$. Also, in vitro experiments demonstrated that HO- 1 was markedly promoted by hypoxia in human placental choriocarcinoma JEG-3 cells, 6 or $12 \mathrm{~h}$ post treatment ( $p<0.05$ or $p<0.01$ ). However, the STAT3 phosphorylation (Tyr 705) was attenuated by sustained hypoxia $(p<0.01)$. Moreover, it was demonstrated that HO-1 overexpression significantly inhibited the hypoxia-promoted STAT3 phosphorylation (Tyr 705). Conclusions: HO-1 was overexpressed in PE placenta, in association with reduced STAT3 phosphorylation (Tyr 705). HO-1 inhibits the STAT3 phosphorylation in placental JEG-3 cells under hypoxia. Thus, we speculate that overexpressed HO-1 might contribute to the reduced STAT3 phosphorylation (Tyr 705) and the pathogenesis of preeclampsia.

Key words: heme oxygenase 1, STAT3 phosphorylation (Tyr 705), hypoxia, preeclampsia.

\section{Introduction}

Preeclampsia (PE) is a pregnancy-related syndrome with hypertension and proteinuria after the $20^{\text {th }}$ week of gestation, affecting $3-5 \%$ of pregnancies worldwide and causing $15-46 \%$ maternal mortality $(50,000$

\author{
Corresponding author: \\ Xian-Zhen Pan PhD \\ Department of Obstetrics \\ and Gynecology \\ the Affiliated Yantai \\ Yuhuangding Hospital \\ of Qingdao University \\ 20 Yuhuangding Road \\ Zhifu District \\ 264000 Yantai, China \\ Phone: 00865356691999 \\ E-mail: pan_xianzhen@163. \\ com
}


maternal deaths annually) [1-3]. Preeclampsia is currently believed to be caused by inadequate trophoblast invasion and placentation [2]. At the pre-clinical stage of $\mathrm{PE}$, the inadequate trophoblast invasion results in a failure of vascular remodeling and decreased circulation in the early placenta, thus resulting in continuous placental ischemia after 20 weeks of gestation [4, 5]. Increased trophoblast cell apoptosis in the placenta may be the direct cause of PE [6-8]. Widespread apoptosis of placental cytotrophoblasts within the uterine wall was found to be associated with PE [7]. Enhanced trophoblast cell apoptosis in preeclampsia has also been confirmed in vitro $[9,10]$. Moreover, the increased trophoblast apoptosis can decrease trophoblast invasion.

Multiple lines of evidence indicate the involvement of hypoxia in preeclampsia. There is a $50 \%$ reduction in uteroplacental circulation in patients with preeclampsia [11], and there is an increased incidence of preeclampsia in women residing at high altitudes [12]. Also the involvement of hypoxia in preeclampsia was supported by molecular evidence [13] and was confirmed in models of uteroplacental ischemia in rodents and primates $[14,15]$. It is widely believed that the reduced trophoblast invasion into the maternal decidua and the lack of spiral artery remodeling [16] lead to uteroplacental hypoxia in preeclampsia. However, the molecular mechanisms underlying the association of placental hypoxia with preeclampsia are not clear. Increased soluble fms-like tyrosine kinase-1 (sFLT-1) and endothelin-1 have been found in a primate preeclampsia model [15]. Reduced matrix metalloproteinase (MMP)-2 activation has also been recognized in the hypoxia-inhibited invasion of extravillous trophoblast cells [17]. Particularly, STAT3 is indicated to promote trophoblast invasion [18], via regulating the transcription of target genes, such as apoptosis-associated genes Mcl-1L, Bcl-xL, survivin, and invasion-related genes MMP-2 and MMP-9 [19-21]. STAT3-deficient embryos showed rapid degeneration, suggesting that STAT3 is essential for the early development of mouse embryos [22]. Moreover, a previous study found that hypoxia exacerbated impairment of PIGF/JAK-STAT3 signaling in preeclampsia [23]. However, little is known about the regulation of STAT3 activation in preeclampsia.

STAT3 is activated by the entire family of interleukin (IL)-6-type cytokines, through gp130 and related receptors, whose dimerization can subsequently result in the activation of receptor kinase activity. The activated receptor then phosphorylates and activates the Janus tyrosine kinases (JAKs), which subsequently activate STAT3 phosphorylation [24, 25]. Also reduced levels of maternal serum S100-B, PAPP-A and IL- 6 have been observed in severe preeclampsia [26, 27]. However, other studies revealed an opposite profile of proinflammatory cytokines in preeclampsia pregnancies. A higher systemic inflammatory status, including elevated levels of tumor necrosis factor- $\alpha$ (TNF- $\alpha$ ), interleukin-6 (IL-6), and C-reactive protein (CRP), has been observed in preeclampsia patients $[28,29]$. Also there was a higher IL-6 level in monocyte cultures exposed to preeclampsia plasma [30]. Therefore, the mechanisms mediating the pathogenesis of preeclampsia are far from clear. In particular, other biomarkers might be involved in the regulation of STAT3 phosphorylation.

The present study focused on the role in preeclampsia of heme oxygenase $1(\mathrm{HO}-1)$, which is an inducible isoform of $\mathrm{HO}$, in response to stress such as oxidative stress, hypoxia, heavy metals, cytokines, etc. [31]. We examined the expression of HO-1 and the expression and phosphorylation (Tyr705) of STAT3 in the placentas from preeclampsia patients. Then we investigated the regulation by $\mathrm{HO}-1$ of the expression and phosphorylation of STAT3 in a human placental choriocarcinoma JEG-3 cell line under hypoxia. The present study suggests that reduced STAT3 phosphorylation might be associated with the hypoxia-promoted $\mathrm{HO}-1$ in PE.

\section{Material and methods}

\section{Subjects}

Twenty-five preeclamptic pregnant subjects and 20 normal pregnant subjects were recruited at the moment of admission to the Department of Obstetrics and Gynecology, the Affiliated Yantai Yuhuangding Hospital of Qingdao University. The diagnosis of PE was made as previously described [32]. Pregnant subjects with more than 20 weeks of gestation were included when systolic/diastolic blood pressure $\geq 140 / 90 \mathrm{~mm} \mathrm{Hg}$ on 2 separate readings, proteinuria measurement of $1+$ or more on a dipstick, or 24-hour urine protein collection with $\geq 300 \mathrm{mg}$ in the specimen. Subjects with hypertension before pregnancy or before the $20^{\text {th }}$ week of gestation, or with disorders such as chronic renal diseases, diabetes, lupus, and urinary tract infection, were excluded from this study. Normal pregnant subjects with normal blood pressure ( $\leq 140 / 90 \mathrm{~mm} \mathrm{Hg}$ ), no proteinuria, and an absence of obstetric and medical complications were included. Subjects with multiple pregnancy $(\geq 2)$ were also excluded. No significant difference in age or body weight index was observed between the two groups of subjects (Table I). Signed consent was obtained at the time of enrollment, and the ethics committee of our hospital approved this study. 
Table I. Clinical characteristics of pregnant women in the PE and normal control groups

\begin{tabular}{|c|c|c|c|}
\hline Characteristics & $\operatorname{PE}(n=25)$ & Normal $(n=20)$ & $P$-value \\
\hline Maternal age [years] & $32.46 \pm 7.65$ & $28.15 \pm 5.25$ & $>0.05$ \\
\hline Body weight index $\left[\mathrm{kg} / \mathrm{m}^{2}\right]$ & $26.36 \pm 3.92$ & $25.74 \pm 3.66$ & $>0.05$ \\
\hline Gestation [days] & $233.53 \pm 4.87$ & $274.16 \pm 2.48$ & $<0.001$ \\
\hline Diastolic pressure $[\mathrm{mm} \mathrm{Hg}]$ & $109.36 \pm 3.64$ & $71.17 \pm 3.43$ & $<0.001$ \\
\hline Systolic pressure [mm Hg] & $172.28 \pm 8.54$ & $108.53 \pm 3.76$ & $<0.001$ \\
\hline Proteinuria positive (more than + ) & 25 & 0 & - \\
\hline \multicolumn{4}{|l|}{ PE onset: } \\
\hline Early-onset & 16 & - & - \\
\hline Late-onset & 9 & - & - \\
\hline Delivery mode: & & & $<0.001$ \\
\hline Vaginal delivery & 3 & 15 & \\
\hline Cesarean section & 22 & 5 & \\
\hline
\end{tabular}

\section{Cell culture and treatment}

A human placental choriocarcinoma JEG-3 cell line was purchased from American Type Culture Collection (ATCC) (Rockville, MD, USA), and was cultured in Eagle's Minimum Essential Medium (EMEM) (Thermo Fisher Scientific, Inc., Waltham, MA, USA) which were supplemented with $10 \%$ Fetal Bovine Serum (FBS) (Hyclone, Pittsburgh, PA, USA) and with $50 \mu \mathrm{g} / \mathrm{ml}$ penicillin and with $50 \mathrm{\mu g} / \mathrm{ml}$ streptomycin (CSPC Pharmaceutical Group, Shijiazhuang, China). For the cell culture under normoxia, cells were incubated at $37^{\circ} \mathrm{C}$ with $5 \% \mathrm{CO}_{2}$ in a humid incubator. For the hypoxia treatment, JEG-3 cells were placed in a hypoxia incubator infused with a gas mixture of $5 \% \mathrm{CO}_{2}$, $2 \%$ oxygen and nitrogen for $3,6,12,24$ or $48 \mathrm{~h}$. To overexpress HO- 1 in JEG-3 cells, the human HO- 1 coding sequence was amplified and cloned into a pcDNA3.1(+) vector (Invitrogen, Carlsbad, CA, USA). The enhanced green fluorescence protein (EGFP) coding sequence was also cloned into the pcDNA3.1(+) vector as a negative control. HO-1pcDNA3.1(+) or control EGFP-pcDNA3.1(+) plasmids were transfected into JEG-3 cells with Lipofectamine 2000 (Invitrogen, Carlsbad, CA, USA).

\section{Immunofluorescence}

Sections of placentas obtained from normal pregnant females and preeclamptic pregnancies post delivery were fixed with $10 \%$ formalin and were embedded with paraffin. Sliced tissue which was transferred to slides was deparaffinized and stained using a general standard protocol. Polyclonal rabbit anti-STAT3 with or without phosphorylation (Tyr 705) (Abcam, Cambridge, UK), or anti-HO-1 (Abcam, Cambridge, UK) and HRP-cou- pled goat anti-rabbit IgG antibodies (Sino Biological, Beijing, China) were used to detect the STAT3 with or without phosphorylation (Tyr 705) or HO-1 antigens in the placental tissues. HRP substrate 3-amino-9-ethylcarbazole (AEC) (Abcam, Cambridge, UK) was used for staining. The immunostaining was semiquantified by grading the staining proportion and staining density.

\section{RNA extraction and quantitative real-time polymerase chain reaction}

QuantiTect SYBR Green PCR Kits (Qiagen, $\mathrm{GmbH}$, Hilden, Germany) were used to quantify the mRNA level of HO-1 and STAT3 via the quantitative real-time polymerase chain reaction ( $q R T$ PCR) method. The qRT-PCR was performed on a LightCycler 2.0 (Roche Diagnostics, Mannheim, Germany) detection system with the following cycling parameters: $95^{\circ} \mathrm{C}$ for $5 \mathrm{~min}$, followed by 40 cycles of $95^{\circ} \mathrm{C}$ for $15 \mathrm{~s}$ and $60^{\circ} \mathrm{C}$ for $30 \mathrm{~s}$. Primers for HO-1, STAT3 and $\beta$-actin were synthesized by the Sangon company (Shanghai, China). All data are presented as fold change over the internal control ( $\beta$-actin), and were calculated using the $\Delta \Delta$ Ct method.

\section{Western blot analysis of HO-1 and STAT3}

Protein samples were extracted from placental tissues or JEG-3 cells with NE-PER Nuclear and Cytoplasmic Extraction Reagents Kit (Pierce, Rockford, IL, USA). These protein samples were separated by electrophoresis using a $12 \%$ SDS-PAGE gel and then were transferred to a polyvinylidene fluoride hydrophobic membrane (Millipore, Bedford, MA, USA). Then the membrane was blocked with 2\% BSA (Sigma-Aldrich, St. Louis, MO, USA) 
overnight at $4^{\circ} \mathrm{C}$ and was incubated with rabbit polyclonal antibody against human $\mathrm{HO}-1$ and STAT3 with or without phosphorylation (Tyr 705) or $\beta$-actin respectively. Finally, HRP-conjugated secondary antibody against rabbit IgG and electrochemiluminescence (ECL) (Amersham, Uppsala, Sweden) were used to visualize the protein bands. The levels of HO-1 and STAT3 with or without phosphorylation (Tyr 705) were presented as a percent gray value normalized to $\beta$-actin.

\section{Statistical analysis}

Quantitative data were presented as means \pm SEM. A nonparametric Mann-Whitney test or ANOVA StatView (Cary, NC) was used for data analysis. Statistical significance was considered when $p<0.05$ or less.

\section{Results}

\section{Downregulated phosphorylation of STAT3 in PE placental specimens}

To investigate the STAT3 activation and its regulatory role in placental tissues from preeclamptic subjects, we examined the expression and phosphorylation (Tyr 705) of STAT3 in 25 preeclamptic placental villous specimens and in 20 normal puerperae with the immunohistochemical method. It was demonstrated that STAT3 was expressed to a high level in the placental tissues between the normal or PE groups (Figures $1 \mathrm{~A}, \mathrm{~B}$ ); however, the STAT3-positive cells were not significantly different in PE placental specimens between the normal and PE groups ( $p>0.05$, Figure $1 \mathrm{C})$. As indicated in Figures $1 \mathrm{D}$ and $\mathrm{E}$, the phosphorylated STAT3 level was significantly lower in the PE group than in the normal group $(p<0.01$, Figure $1 \mathrm{~F})$. Then we re-evaluated the STAT3 expression in both protein and mRNA levels with western blotting and RT-qPCR methods. It can be observed in Figure $2 \mathrm{~A}$ that the protein level of STAT3 was lower in the PE placental tissues than in normal placental tissues, although the reduction was not significant ( $p>0.05$, Figure 2 B). However, the relative level of phosphorylated (Tyr 705) form of STAT3 was markedly lower in the PE group $(p<0.01$, Figure $2 \mathrm{C}$ ). In addition, the STAT3 mRNA level was not significantly different between the two groups ( $p=0.4429$, Figure $2 \mathrm{D})$.

\section{Upregulation of $\mathrm{HO}-1$ in preeclampsia placental specimens}

To investigate the $\mathrm{HO}-1$ expression in preeclamptic placental tissues, we also examined the HO-1 expression in the placental villous specimens from both groups. Firstly, the HO-1 protein level was analyzed with immunohistochemistry, and it can be observed in Figure $3 \mathrm{~A}$ (normal) and $3 \mathrm{~B}(\mathrm{PE})$ that there were more $\mathrm{HO}$-1-positive trophoblast cells in the PE group, according to the staining intensity and staining area. Then a western blotting assay was performed to re-evaluate the HO-1 protein level in both groups. As shown in Figure $3 \mathrm{C}$ (normal) and $3 \mathrm{D}(\mathrm{PE})$, the protein level of HO-1 was markedly higher in the PE group than in the normal group $(p<0.001)$. In addition, we examined the mRNA level of HO-1 with RT-qPCR in the normal and PE groups. Figure $3 \mathrm{E}$ demonstrates that the HO-1 mRNA level was also markedly higher in the high PE group $(p<0.0001)$. Therefore, we confirmed the upregulation of HO-1 in the placental villous specimens of PE patients.

\section{Hypoxia upregulates $\mathrm{HO}-1$ expression and STAT3 activation in human placental choriocarcinoma JEG-3 cells}

Hypoxia has been indicated to be a contributor to the pathogenesis of preeclampsia $[33,34]$. To elucidate a possible role of hypoxia in the $\mathrm{HO}-1$ upregulation and the reduced STAT3 phosphorylation in PE placental tissues, we selected a human placental choriocarcinoma JEG-3 cell line, to evaluate the expression of HO-1 and STAT3 and the activation of STAT3 in such cells under hypoxia. Firstly, as shown in Figure $4 \mathrm{~A}$, the mRNA level of HO-1 was significantly upregulated in the JEG-3 cells, post hypoxia, for 6 to $48 \mathrm{~h}$ after hypoxia treatment $(p<0.05$ or $p<0.01)$. The upregulation of HO-1 was also confirmed via the western blotting assay; the protein level of $\mathrm{HO}-1$ was markedly higher in the hypoxia-treated JEG-3 cells ( $p<0.05$, $p<0.01$ or $p<0.001$, Figures $4 \mathrm{~B}$ and C), with a time dependence $(p<0.01)$. It was indicated that the STAT3 expression was not markedly regulated by the hypoxia treatment in either mRNA (Figure $4 \mathrm{D}$ ) or protein (Figures $4 \mathrm{E}$ and F) levels. However, the relative level of phosphorylated STAT3 (Tyr 705) to STAT3 was significantly promoted by hypoxia at 12,24 or $48 \mathrm{~h}$ post treatment $(p<$ $0.01, p<0.001$ and $p<0.05$ respectively for 12 , 24 and $48 \mathrm{~h}$ post treatment, Figures $4 \mathrm{E}$ and $\mathrm{G}$ ). Interestingly, the level of phosphorylated STAT3 (Tyr 705) was markedly lower at 48 than at $24 \mathrm{~h}$ post treatment ( $p<0.01$ for column 4 vs. column 3 in Figures $4 \mathrm{E}$ and $\mathrm{G}$ ). Thus, we confirmed the upregulation of HO-1 expression and the STAT3 phosphorylation by hypoxia in JEG-3 cells.

Overexpressed HO-1 limits the hypoxiainduced STAT3 phosphorylation in JEG-3 cells

HO- 1 has been confirmed to inhibit the STAT3 phosphorylation in ovalbumin-induced neutrophilic airway inflammation [35] and in endothelial cells [36]. To associate the $\mathrm{HO}-1$ upregulation 

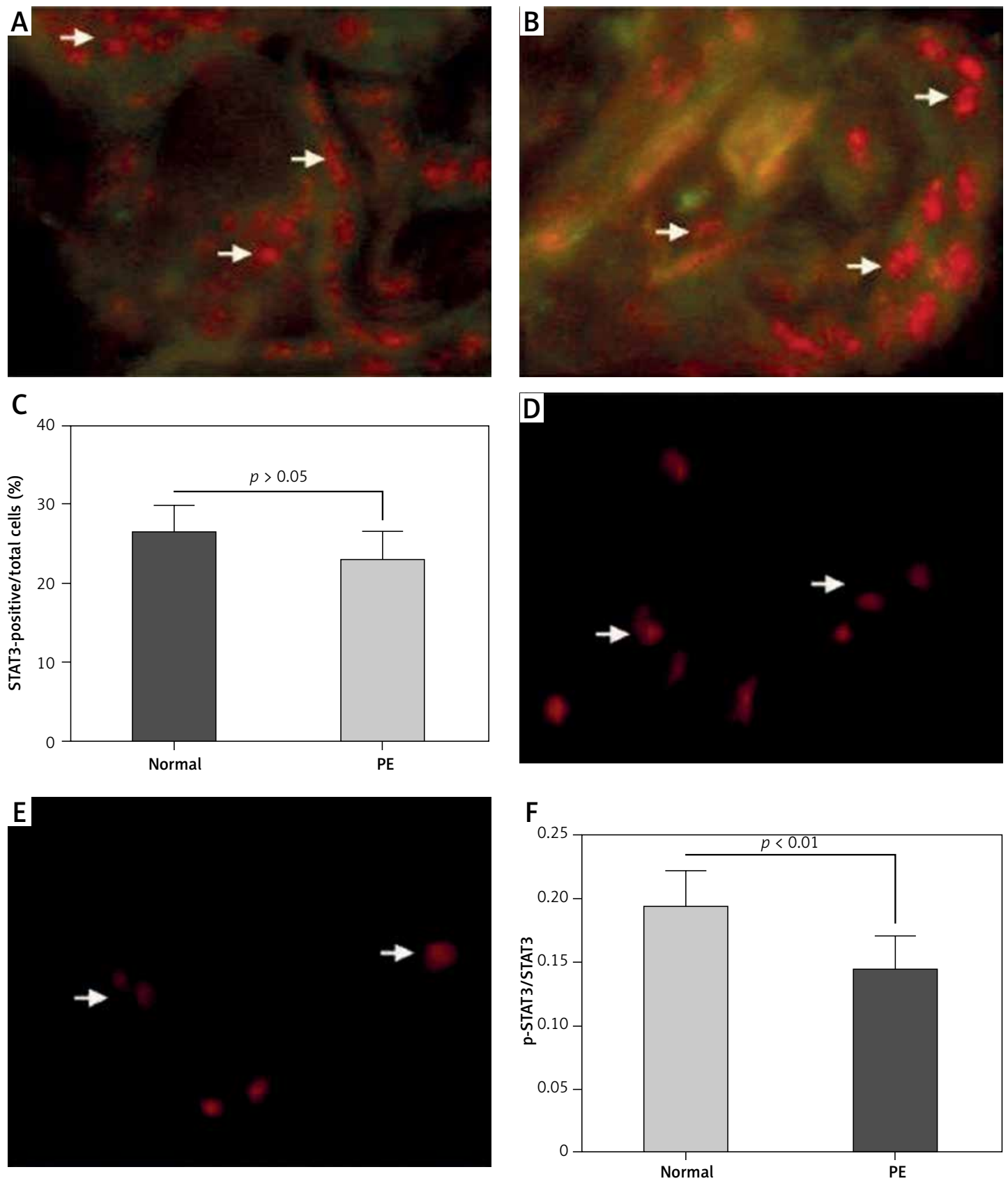

Figure 1. Immunohistochemical analysis of STAT3 with or without Tyr 705 phosphorylation in preeclampsia (PE) placental specimens. STAT3 expression and STAT3 phosphorylation (Tyr 705) were assayed by immunofluorescence staining on placenta tissues from preeclampsia (PE) $(n=25)$ or from normal $(n=20)$ subjects. Red color indicates antibody staining for STAT3 expression or phosphorylation (Tyr 705). A and B - Immunofluorescence staining for STAT3 expression in normal subjects (A) and in PE subjects (B); C - counting for STAT3-positive cells in both groups; D and $\mathbf{E}$ - staining for STAT3 phosphorylation (Tyr 705) in normal subjects (D) and in PE subjects (E); F - ratio of p-STAT3(Tyr 705)-positive to STAT3-positive cells in both groups

with the STAT3 phosphorylation in PE tissues and in JEG-3 cells, we then overexpressed HO-1, and then re-evaluated the STAT3 phosphorylation in the JEG-3 cells, which were subject to hypoxia. It was demonstrated that $\mathrm{HO}-1$ was significantly promoted in both mRNA $(p<0.001$ respectively for 12 or $24 \mathrm{~h}$ post transfection, Figure $5 \mathrm{~A}$ ) and protein ( $p<0.01$ or $p<0.001$, Figure 5 B) levels. However, the HO-1 overexpression did not cause a significant change in the viability of JEG-3 cells (Figure 5 C) under normoxia. Then we re-evaluated the expression and phosphorylation of STAT3 in the JEG-3 cells under hypoxia. It was demonstrated that the HO-1 overexpression did not regulate the STAT3 mRNA level in JEG-3 cells at either 12 or $24 \mathrm{~h}$ post transfection (Figure $5 \mathrm{D}$ ). Also the protein level of STAT3 was not significantly different between the control and HO-1(+) JEG-3 cells 
A

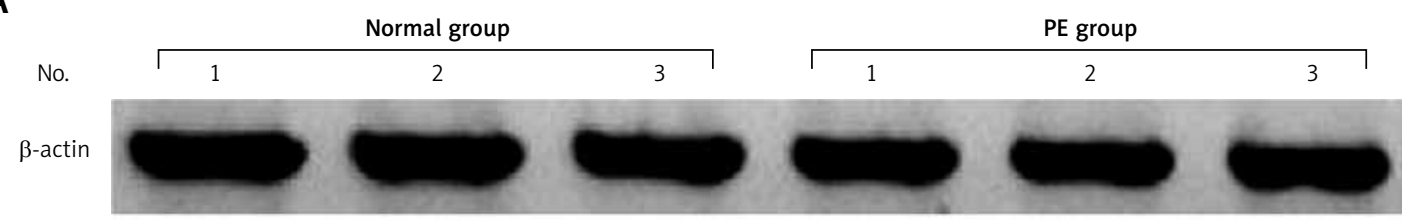

STAT3
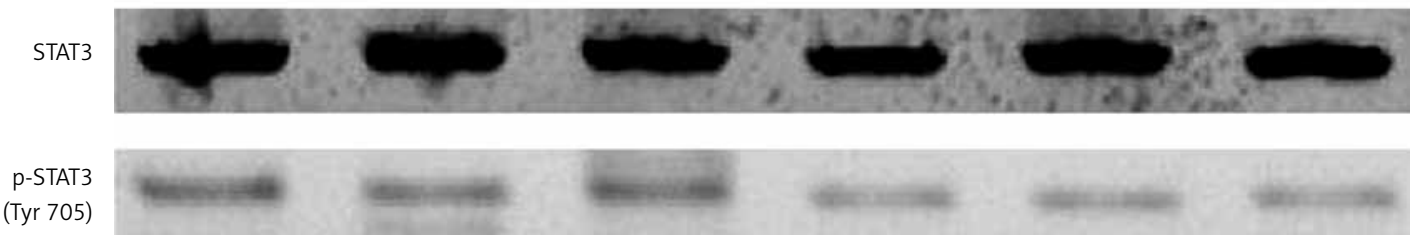

B

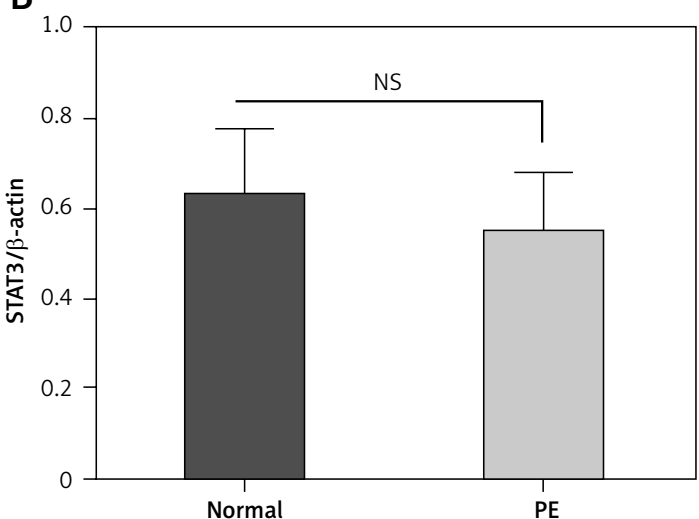

D

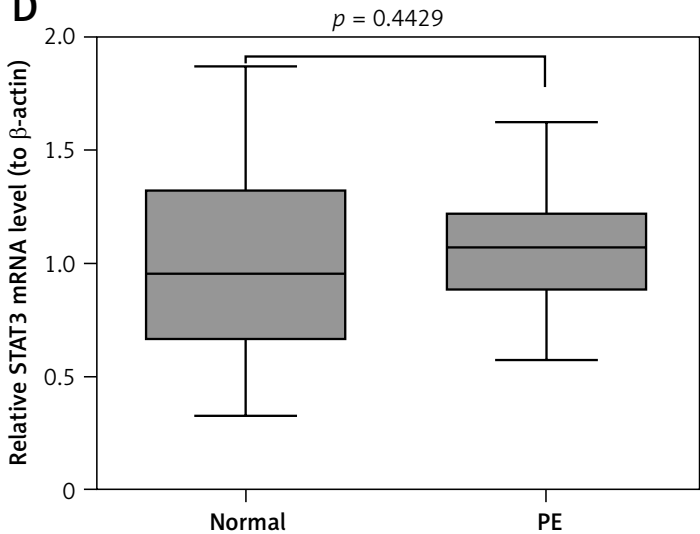

(Figure $5 \mathrm{E}$ ). However, there was a lower level of phosphorylated STAT3 in the HO-1(+) JEG-3 cells than in control JEG-3 cells $(p<0.05$ or $p<0.01$, Figures $5 \mathrm{E}$ and $\mathrm{F}$ ), under hypoxia. Therefore, the overexpressed HO-1 limits the hypoxia-induced STAT3 phosphorylation in JEG-3 cells.

\section{Discussion}

Trophoblast-mediated implantation and placentation are essential steps for pregnancy and the future development of the embryo [37, 38]. The trophoblast cell proliferation initially forms the ectoplacental cone, cells from which then differentiate to form the labyrinth, spongiotrophoblast layer and giant cell compartment $[39,40]$.

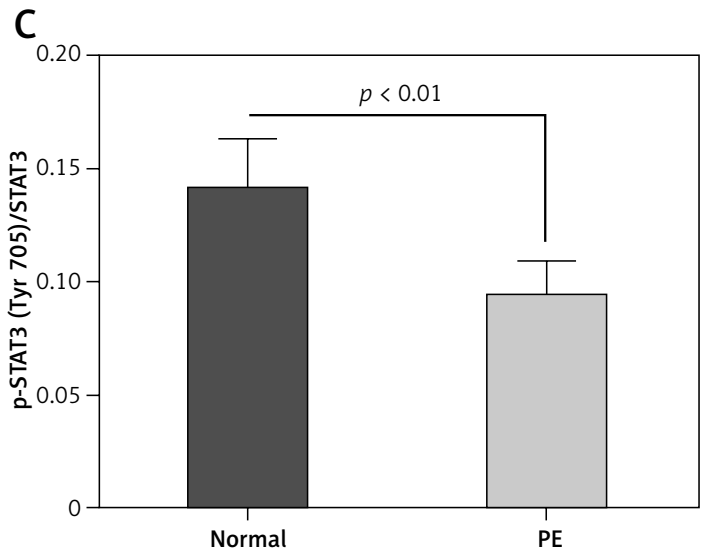

Figure 2. STAT3 expression and phosphorylation (Tyr 705) in preeclampsia (PE) placental specimens, with western blotting assay and RT-qPCR. A - representative western blotting assay results of STAT3 with or without phosphorylation (Tyr 705) in the placenta tissues from PE $(n=25)$ or from normal $(n=20)$ subjects; $\mathbf{B}$ - relative protein band intensities of STAT3 to $\beta$-actin in the placenta tissues from the two groups; $\mathrm{C}$ - relative protein band intensities of STAT3 with phosphorylation (Tyr 705) to STAT3 without phosphorylation (Tyr 705) in the placenta tissues from the two groups; $\mathbf{D}$ - relative mRNA level of STAT3 to $\beta$-actin in the placenta tissues from the two groups

Recently, STAT signaling has been indicated to regulate trophoblast functions during placentation. A variety of growth factors and cytokines activate STAT3, via inducing its phosphorylation and translocation into the nucleus, and promote the invasiveness of trophoblasts [41-43]. However, little is known about the expression and phosphorylation of STAT3 in situ during placental development. In the present study, we investigated the expression and activation of STAT3 in placental tissues from preeclamptic subjects via the immunohistochemical method, western blotting assay and RT-qPCR method, and found that there was a lower level of STAT3 phosphorylation (Tyr 705) in the preeclamptic placental tissues than in the normal 

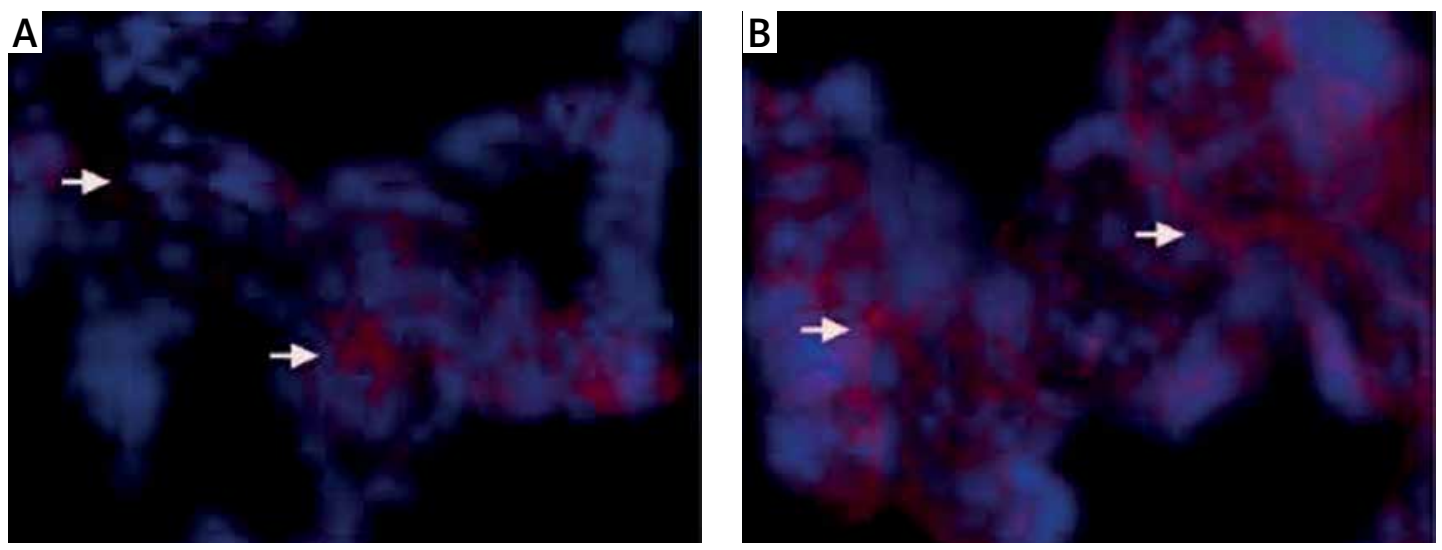

C

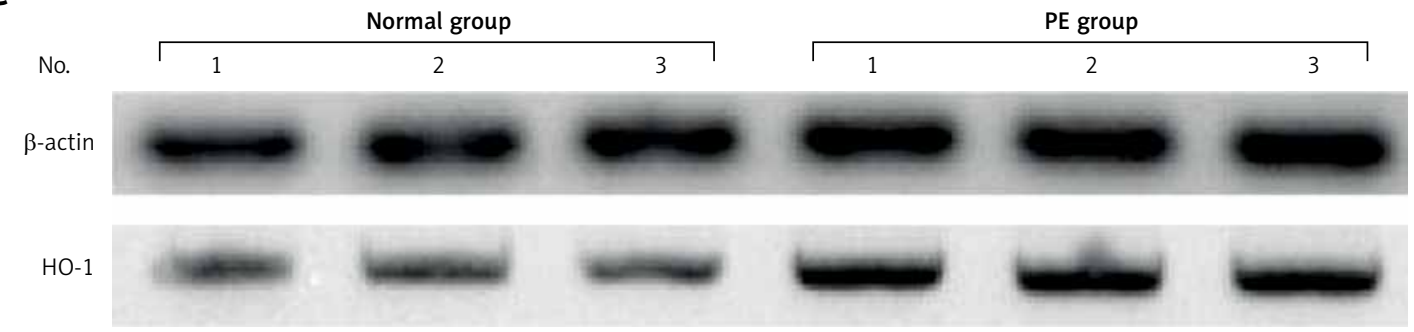

D

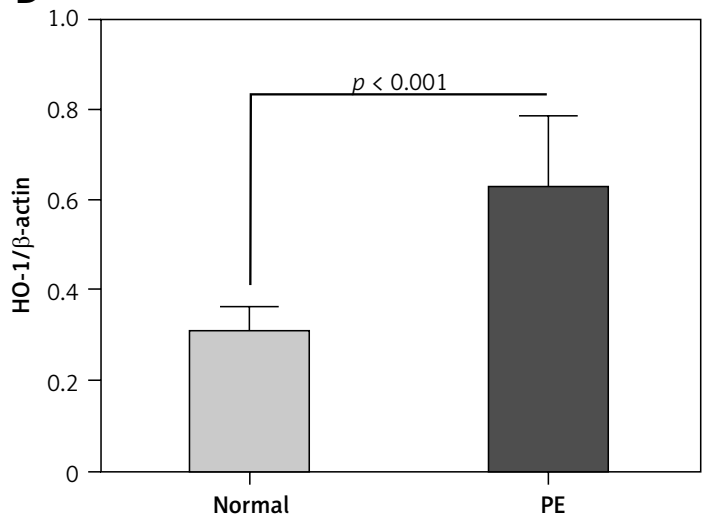

$\mathrm{E}$

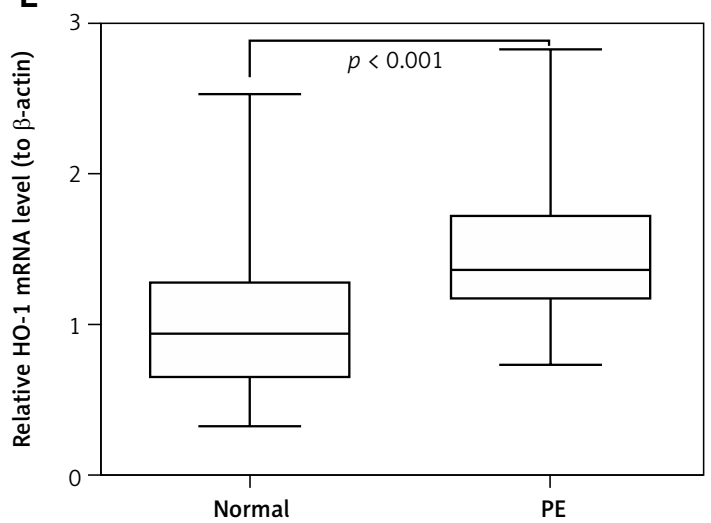

Figure 3. Analysis of HO-1 expression in preeclampsia (PE) placental specimens, with immunohistochemistry, western blotting assay and RT-qPCR. A and B - representative immunofluorescence staining of HO-1 in the placenta tissues from preeclampsia (PE) $(n=25, \mathrm{~A})$ or from normal $(n=20, \mathrm{~B})$ subjects. Red color indicates antibody staining for HO-1 expression. $\mathbf{C}$ and $\mathbf{D}$ - representative western blotting assay image $(C)$ and the relative band intensity of HO- $1 / \beta$-actin in the placenta tissues from PE $(n=25)$ or from normal $(n=20)$ subjects. $\mathrm{E}$ - Relative mRNA level of $\mathrm{HO}-1$ to $\beta$-actin in the placenta tissues from the two groups

placental tissues, implying reduced activation of STAT3 signaling.

STAT3 is activated by the entire family of IL6-type cytokines, via the phosphorylation and activation of JAKs $[24,25]$. Also, reduced levels of maternal serum S100-B, PAPP-A and IL- 6 have been observed in severe preeclampsia [26, 27]. However, other studies have reported an opposite profile of proinflammatory cytokines in preeclampsia pregnancies. A higher systemic inflammatory status, including elevated levels of TNF- $\alpha$, IL-6, and CRP, has been observed in preeclampsia patients $[28,29]$. Also, there was a higher IL-6 level in monocyte cultures exposed to preeclampsia plasma [30]. Therefore, other biomarkers might be involved in the regulation of STAT3 phosphorylation (Tyr 705). Our previous study indicated that hypoxia exacerbated impairment of PIGF/ JAK-STAT3 signaling in preeclampsia [44]. In the present study, we found upregulation of HO-1 in the preeclamptic placental tissues, along with reduced STAT3 phosphorylation (Tyr 705). Also the in vitro experiments demonstrated that $\mathrm{HO}-1$ was markedly promoted by hypoxia in human placental choriocarcinoma JEG-3 cells, time dependently. However, the STAT3 phosphorylation (Tyr 705) was attenuated by the sustained hypoxia (from 24 to $48 \mathrm{~h}$ post treatment), though it was also mark- 
A

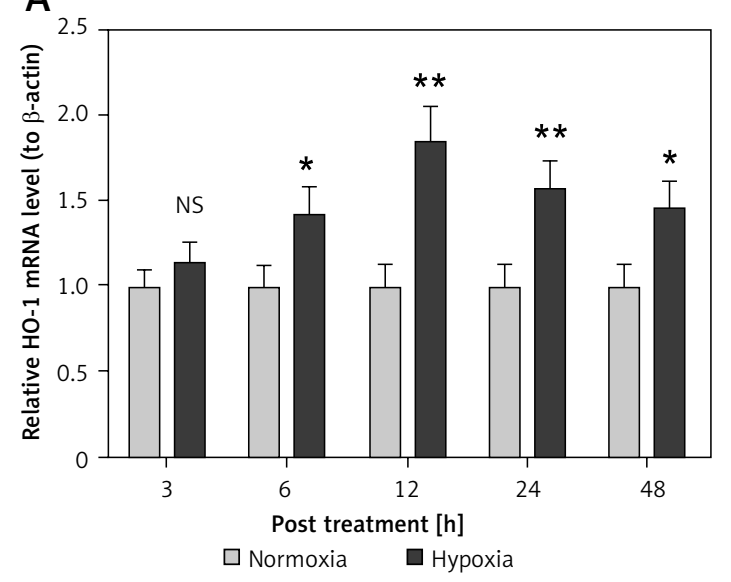

C

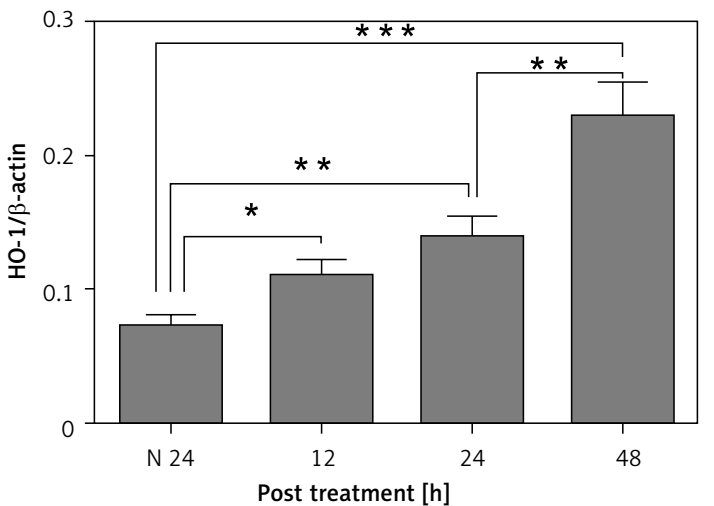

$E$

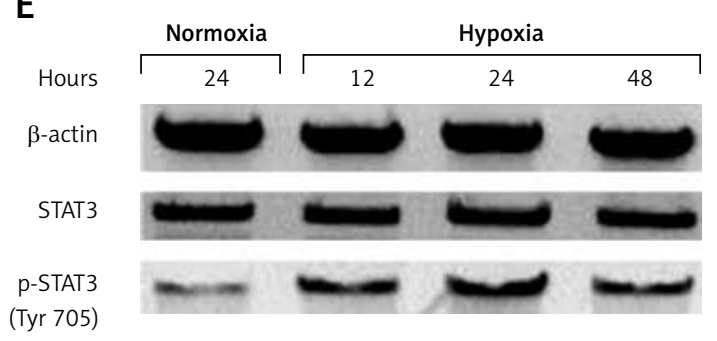

G

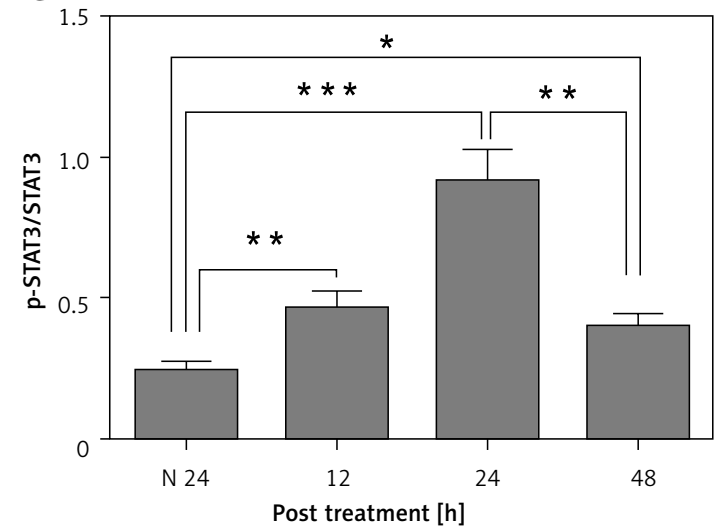

B
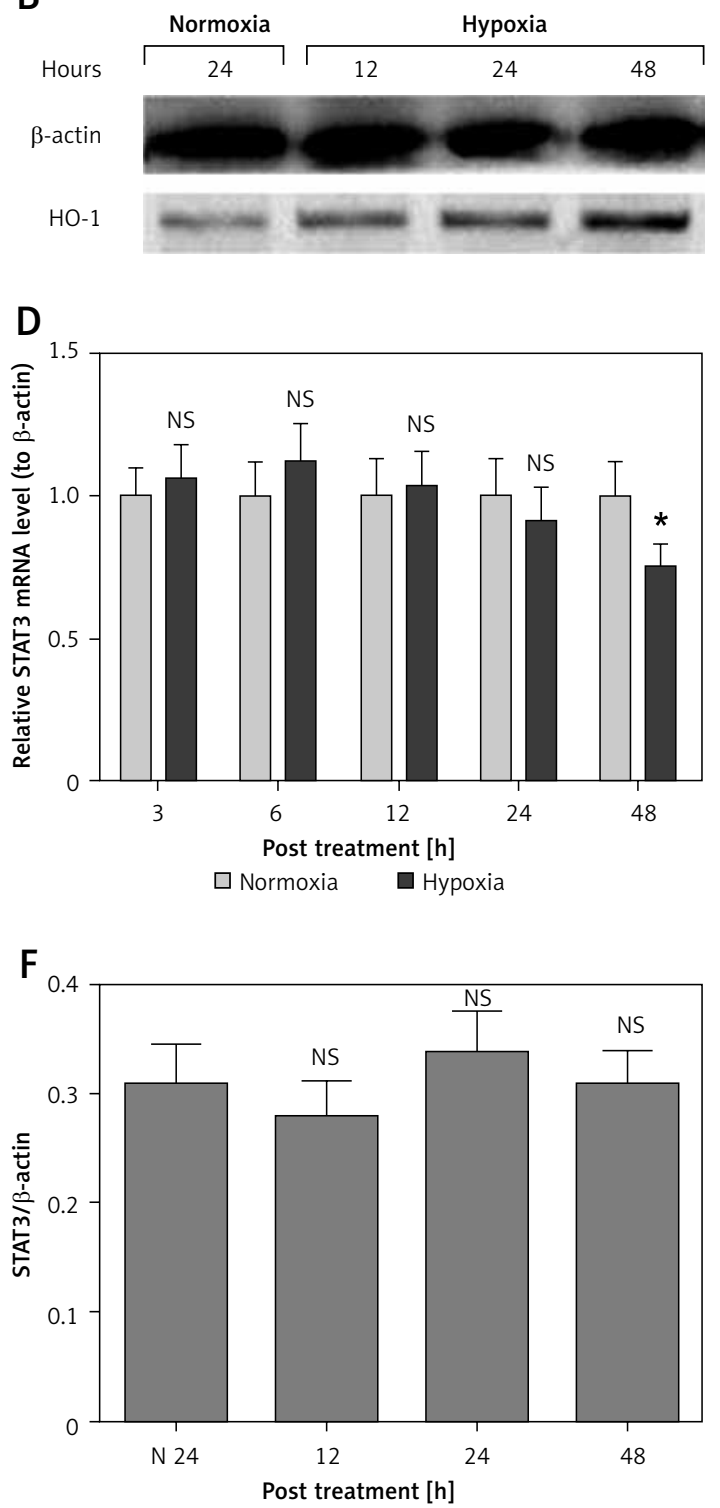

Figure 4. Hypoxia-mediated regulation of HO-1 expression and STAT3 phosphorylation in human placental choriocarcinoma JEG-3 cells. A - Relative mRNA level of HO-1 to $\beta$-actin in the JEG-3 cells under normoxia or hypoxia for $3,6,12,24$ or $48 \mathrm{~h}$; B and C - western blotting assay (B) and the relative band intensity of $\mathrm{HO}-1 / \beta$-actin in the JEG-3 cells under normoxia or hypoxia for 12,24 or $48 \mathrm{~h}$; $\mathbf{D}$ - relative mRNA level of STAT3 to $\beta$-actin in the JEG-3 cells under normoxia or hypoxia for 3,6 , 12, 24 or 48 h; E - western blotting assay of STAT3 with or without phosphorylation (Tyr 705) in the JEG-3 cells under normoxia or hypoxia for 12,24 or 48 h; F and $\mathbf{G}$ - Relative band intensity of STAT3/ $\beta$ actin (F) or phosphorylated STAT3 (Tyr 705)/STAT3 in the JEG-3 cells under normoxia or hypoxia. Data were averaged for triple independent experiments. Asterisks indicate statistical significance $\left({ }^{*} p<0.05\right.$, ${ }^{* *} p<0.01$ or ${ }^{* * *} p<0.001$ ) 
A

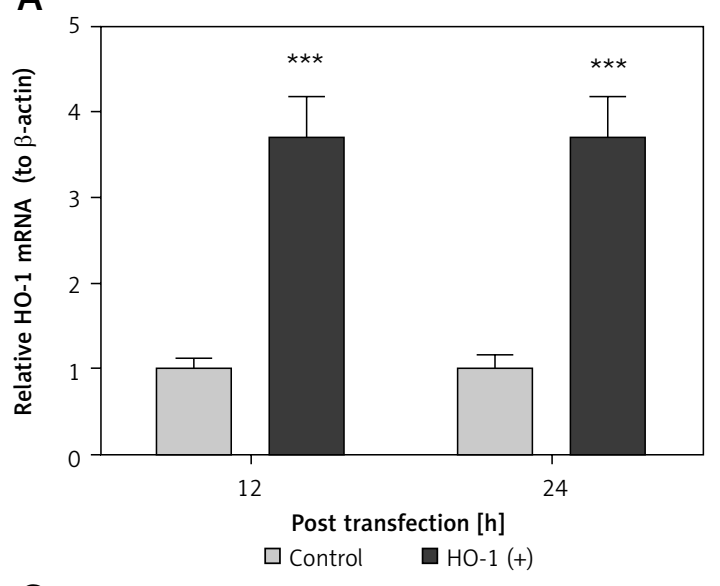

C

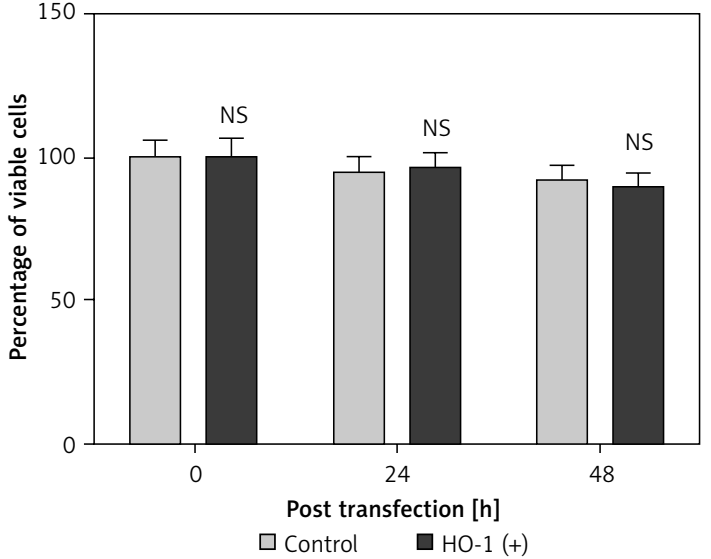

E

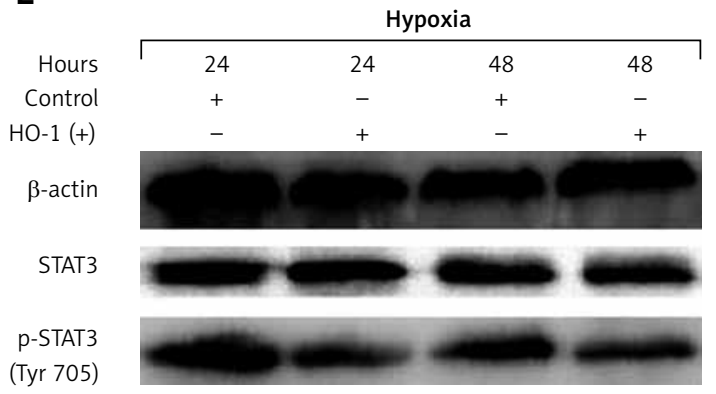

Figure 5. Expression and phosphorylation of STAT3 in the hypoxia-treated JEG-3 cells, post HO-1 overexpression. A - relative mRNA level of $\mathrm{HO}-1$ to $\beta$-actin in the JEG-3 cells, which were transfected with $\mathrm{HO}-1$ pcDNA3.1(+) (HO-1(+)) or with EGFP-pcDNA3.1(+) (Control) for 12 or $24 \mathrm{~h}$; B - Western blotting assay and the relative band intensity of $\mathrm{HO}-1 / \beta$-actin in the control or HO-1(+) JEG-3 cells for 24 or $48 \mathrm{~h}$; C - percent viability of the control or HO-1(+) JEG-3 cells, after incubation for 0,24 or $48 \mathrm{~h} ; \mathrm{D}$ - relative mRNA level of STAT3 to $\beta$-actin in the JEG-3 cells, which were transfected with HO-1-pcDNA3.1(+) (HO-1(+)) or with EGFP-pcDNA3.1(+) (Control) for 0, 12 or $24 \mathrm{~h} ; \mathrm{E}$ and $\mathbf{F}$ - western blotting assay (E) and the relative band intensity (F) of phosphorylated STAT3/STAT3 in the control or HO-1(+) JEG-3 cells, after hypoxia treatment for 24 or $48 \mathrm{~h}$. Data were averaged for triple independent experiments. Asterisks indicate statistical significance $\left({ }^{*} p<0.05,{ }^{* *} p<\right.$ 0.01 or $\left.^{* * *} p<0.001\right)$

\section{B} Hours

Control

HO-1 (+)

$\beta$-actin

HO-1
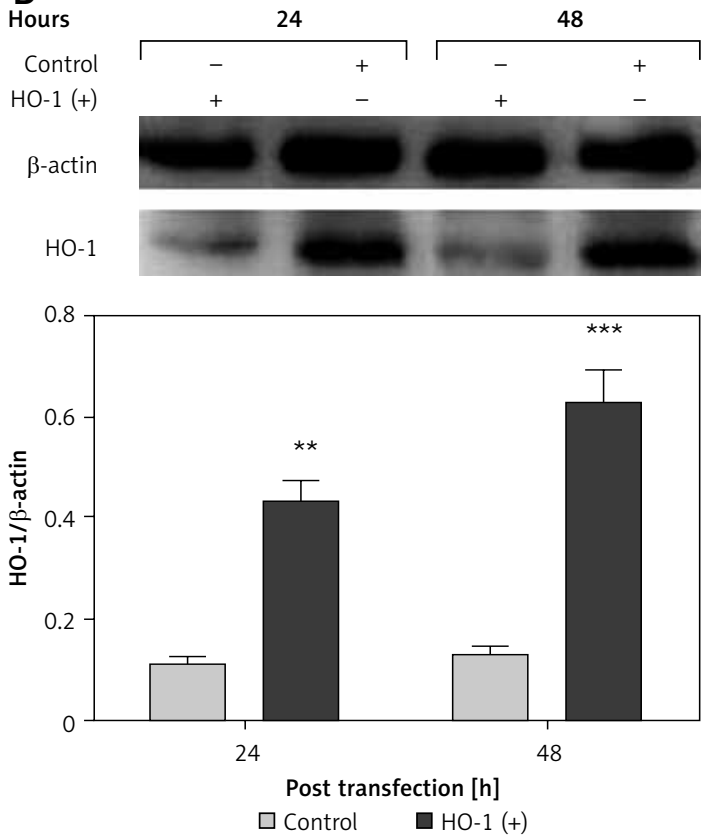

D

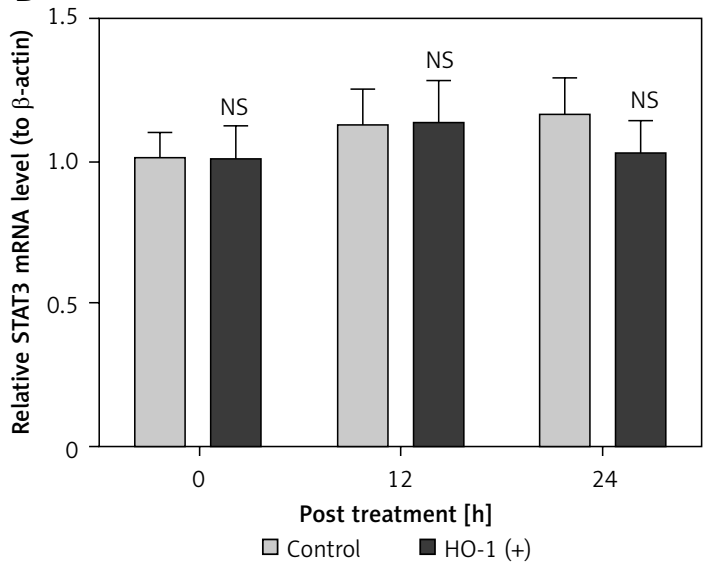

F

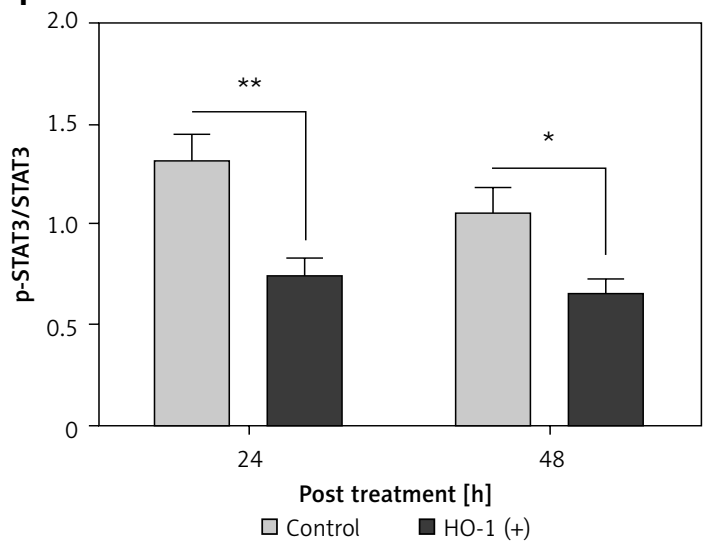


edly promoted by hypoxia in JEG-3 cells. Moreover, the gain-of-function experiment demonstrated that the HO-1 overexpression significantly inhibited the hypoxia-promoted STAT3 phosphorylation (Tyr 705). Thus, we speculated that the HO-1-mediated attenuation of STAT3 phosphorylation (Tyr 705) might contribute to the lower STAT3 phosphorylation (Tyr 705) level in preeclamptic placentas.

Hypoxia activates nuclear factor E2-related factor 2 (Nrf2) and promotes the expression of its target gene, $\mathrm{HO}-1$, which then ameliorates the increased apoptosis and impaired proliferation and angiogenesis in endothelial progenitor cells (EPCs) [45]. The upregulation of $\mathrm{HO}-1$ has been shown to effectively prevent the chemical-induced oxidative damage in BeWo cells (as a model for syncytiotrophoblast formation) [46]. However, another study demonstrated that the early stage of hypoxia-induced oxidative and inflammatory stresses leads to acceleration of cell apoptosis via Nrf2/HO-1 pathways in endothelial cells [47]. There seems to be a positive feedback loop of vascular endothelial growth factor (VEGF) expression and Nrf2/ HO-1 induction [48]. Such a positive feedback loop eventually results in an exhausted VEGF bioavailability, a higher vulnerability to placental oxidative cell damage and finally a vicious circle that may end up in preeclampsia [48]. Our study indicated that the hypoxia-promoted $\mathrm{HO}-1$ also contributes to the impairment of JAK-STAT3 signaling, which might be another mechanism underlying the pathogenesis of preeclampsia. There have been studies supporting the reduced HO-1 expression in preeclamptic placenta [49]. However, other studies found that HO-1 was not decreased in preeclamptic placenta [50]. Interestingly, we found overexpression of $\mathrm{HO}-1$ in the preeclamptic placenta. Therefore, there need to be more studies on $\mathrm{HO}-1$ expression in the preeclamptic placenta.

In conclusion, $\mathrm{HO}-1$ was overexpressed in the preeclamptic placenta, in association with reduced STAT3 phosphorylation (Tyr 705). Also HO-1 inhibits the STAT3 phosphorylation in placental JEG-3 cells under hypoxia. Thus, we speculated that the overexpressed $\mathrm{HO}-1$ might contribute to the lower STAT3 phosphorylation (Tyr 705) and the pathogenesis of preeclampsia.

\section{Acknowledgments}

Hong-Mei Qu and Li-Ping Qu - contributed equally to this work.

The present study was supported by grants from the National Nature Science Foundation of China (No. 81601288).

\section{Conflict of interest}

The authors declare no conflict of interest.

\section{References}

1. Duley L. The global impact of pre-eclampsia and eclampsia. Semin Perinatol 2009; 33: 130-7.

2. Steegers EA, von Dadelszen P, Duvekot JJ, et al. Pre-eclampsia. Lancet 2010; 376: 631-44.

3. Ghulmiyyah L, Sibai B. Maternal mortality from preeclampsia/eclampsia. Semin Perinatol 2012; 36: 56-9.

4. Red-Horse K, Zhou Y, Genbacev O, et al. Trophoblast differentiation during embryo implantation and formation of the maternal-fetal interface. J Clin Invest 2004; 114: 744-54.

5. Genbacev O, Zhou Y, Ludlow JW, et al. Regulation of human placental development by oxygen tension. Science 1997; 277: 1669-72.

6. Davison JM, Homuth V, Jeyabalan A, et al. New aspects in the pathophysiology of preeclampsia. J Am Soc Nephrol 2004; 15: 2440-8

7. DiFederico E, Genbacev O, Fisher SJ. Preeclampsia is associated with widespread apoptosis of placental cytotrophoblasts within the uterine wall. Am J Pathol 1999; 155: 293-301.

8. Ishihara N, Matsuo $\mathrm{H}$, Murakoshi $\mathrm{H}$, et al. Increased apoptosis in the syncytiotrophoblast in human term placentas complicated by either preeclampsia or intrauterine growth retardation. Am J Obstet Gynecol 2002; 186: 158-66.

9. Crocker IP, Cooper S, Ong SC, et al. Differences in apoptotic susceptibility of cytotrophoblasts and syncytiotrophoblasts in normal pregnancy to those complicated with preeclampsia and intrauterine growth restriction. Am J Pathol 2003; 162: 637-43.

10. Hu C, Smith SD, Pang L, et al. Enhanced basal apoptosis in cultured term human cytotrophoblasts is associated with a higher expression and physical interaction of $\mathrm{p} 53$ and Bak. Placenta 2006; 27: 978-83.

11. Lunell NO, Nylund LE, Lewander R, et al. Uteroplacental blood flow in pre-eclampsia measurements with indium-113m and a computer-linked gamma camera. Clin Exp Hypertens B 1982; 1: 105-17.

12. Zamudio $S$, Wu Y, letta F, et al. Human placental hypoxia-inducible factor-1alpha expression correlates with clinical outcomes in chronic hypoxia in vivo. Am J Pathol 2007; 170: 2171-9.

13. Soleymanlou N, Jurisica I, Nevo O, et al. Molecular evidence of placental hypoxia in preeclampsia. J Clin Endocrinol Metab 2005; 90: 4299-308.

14. Gilbert J, Dukes M, LaMarca B, et al. Effects of reduced uterine perfusion pressure on blood pressure and metabolic factors in pregnant rats. Am J Hypertens 2007; 20: 686-91.

15. Makris A, Thornton C, Thompson J, et al. Uteroplacental ischemia results in proteinuric hypertension and elevated sFLT-1. Kidney Int 2007; 71: 977-84.

16. Meekins JW, Pijnenborg R, Hanssens M, et al. A study of placental bed spiral arteries and trophoblast invasion in normal and severe pre-eclamptic pregnancies. $\mathrm{Br} J \mathrm{Ob}$ stet Gynaecol 1994; 101: 669-74.

17. Onogi A, Naruse K, Sado T, et al. Hypoxia inhibits invasion of extravillous trophoblast cells through reduction of matrix metalloproteinase (MMP)-2 activation in the early first trimester of human pregnancy. Placenta 2011; 32: 665-70.

18. Fitzgerald JS, Poehlmann TG, Schleussner E, et al. Trophoblast invasion: the role of intracellular cytokine signalling via signal transducer and activator of transcription 3 (STAT3). Hum Reprod Update 2008; 14: 335-44. 
19. Garcia MG, Tirado-Gonzalez I, Handjiski B, et al. High expression of survivin and down-regulation of Stat-3 characterize the feto-maternal interface in failing murine pregnancies during the implantation period. Placenta 2007; 28: 650-7.

20. Li CF, Gou WL, Li XL, et al. Reduced expression of survivin, the inhibitor of apoptosis protein correlates with severity of preeclampsia. Placenta 2012; 33: 47-51.

21. Xie TX, Wei D, Liu M, et al. Stat3 activation regulates the expression of matrix metalloproteinase- 2 and tumor invasion and metastasis. Oncogene 2004; 23: 3550-60.

22. Takeda K, Noguchi K, Shi W, et al. Targeted disruption of the mouse Stat3 gene leads to early embryonic lethality. Proc Natl Acad Sci U S A 1997; 94: 3801-04.

23. Zhang Z, Yang X, Zhang L, et al. Decreased expression and activation of Stat3 in severe preeclampsia. J Mol Histol 2015; 46: 205-19.

24. Hirano T, Ishihara K, Hibi M. Roles of STAT3 in mediat ing the cell growth, differentiation and survival signals relayed through the IL- 6 family of cytokine receptors. Oncogene 2000; 19: 2548-56

25. Huang WL, Yeh HH, Lin CC, et al. Signal transducer and activator of transcription 3 activation up-regulates interleukin- 6 autocrine production: a biochemical and genetic study of established cancer cell lines and clinical isolated human cancer cells. Mol Cancer 2010; 9: 309.

26. Artunc-Ulkumen B, Guvenc Y, Goker A, et al. Maternal Serum S100-B, PAPP-A and IL-6 levels in severe preeclampsia. Arch Gynecol Obstet 2015; 292: 97-102.

27. Chen Q, Zhao M, Guo F, et al. The reduction of circulating levels of IL-6 in pregnant women with preeclampsia by magnesium sulphate and nifedipine: in vitro evidence for potential mechanisms. Placenta 2015; 36: 661-6.

28. Mihu D, Razvan C, Malutan A, et al. Evaluation of maternal systemic inflammatory response in preeclampsia. Taiwan J Obstet Gynecol 2015; 54: 160-6.

29. Udenze I, Amadi C, Awolola N, et al. The role of cytokines as inflammatory mediators in preeclampsia. Pan Afr Med J 2015; 20: 219.

30. Rahardjo B, Widjajanto $E$, Sujuti $H$, et al. Different levels of IL-1alpha, IL-6, TNF-alpha, NF-kappaB and PPAR-gamma in monocyte cultures exposed by plasma preeclampsia and normotensive pregnancy. Pregnancy Hypertens 2014; 4: 187-93.

31. Ryter SW, Alam J, Choi AM. Heme oxygenase-1/carbon monoxide: from basic science to therapeutic applications. Physiol Rev 2006; 86: 583-650.

32. Seck K, Fischer T. Diagnosis and treatment of preeclampsia: overview of international recommendations. Z Geburtshilfe Neonatol 2009; 213: 106-12.

33. Iriyama T, Wang W, Parchim NF, et al. Hypoxia-independent upregulation of placental hypoxia inducible factor-1alpha gene expression contributes to the pathogenesis of preeclampsia. Hypertension 2015; 65: 1307-15.

34. Bosco C, Diaz E, Gutierrez R, et al. Placental hypoxia developed during preeclampsia induces telocytes apoptosis in chorionic villi affecting the maternal-fetus metabolic exchange. Curr Stem Cell Res Ther 2016; 11: 420-5.

35. Zhang Y, Zhang L, Wu J, et al. Heme oxygenase-1 exerts a protective role in ovalbumin-induced neutrophilic airway inflammation by inhibiting Th17 cell-mediated immune response. J Biol Chem 2013; 288: 34612-26.

36. Yang YC, Huang YT, Hsieh CW, et al. Carbon monoxide induces heme oxygenase- 1 to modulate STAT3 activa- tion in endothelial cells via S-glutathionylation. Plos One 2014; 9: e100677.

37. Jauniaux E, Burton GJ. Pathophysiology of histological changes in early pregnancy loss. Placenta 2005; 26: 114-23.

38. Norwitz ER. Defective implantation and placentation: laying the blueprint for pregnancy complications. Reprod Biomed Online 2006; 13: 591-9.

39. Muntener M, Hsu YC. Development of trophoblast and placenta of the mouse. A reinvestigation with regard to the in vitro culture of mouse trophoblast and placenta. Acta Anat (Basel) 1977; 98: 241-52.

40. Enders AC, Welsh AO. Structural interactions of trophoblast and uterus during hemochorial placenta formation. J Exp Zool 1993; 266: 578-87.

41. Fitzgerald JS, Busch S, Wengenmayer T, et al. Signal transduction in trophoblast invasion. Chem Immunol Allergy 2005; 88: 181-99.

42. Darnell JJ. Phosphotyrosine signaling and the single cell: metazoan boundary. Proc Natl Acad Sci U S A 1997; 94 : 11767-9.

43. Poehlmann TG, Fitzgerald JS, Meissner A, et al. Trophoblast invasion: tuning through LIF, signalling via Stat3. Placenta 2005; 26 Suppl A: S37-41.

44. Qu HM, Mu LS, Liu HJ, Wang XT. Hypoxia exacerbates the impairment of PIGF/JAK-STAT3 signaling and drug metabolizing enzymes in preeclampsia. Lat Am J Pharm 2016; 35: 17-25.

45. Zhao R, Feng J, He G. Hypoxia increases Nrf2-induced HO-1 expression via the PI3K/Akt pathway. Front Biosci (Landmark Ed) 2016; 21: 385-96.

46. Kweider N, Fragoulis A, Rosen C, et al. Interplay between vascular endothelial growth factor (VEGF) and nuclear factor erythroid 2-related factor-2 (Nrf2): implications for preeclampsia. J Biol Chem 2011; 286: 42863-72.

47. Han Q, Yeung SC, Ip MS, et al. Intermittent hypoxia-induced NF-kappaB and $\mathrm{HO}-1$ regulation in human endothelial EA.hy926 cells. Cell Biochem Biophys 2013; 66: 431-41.

48. Kweider N, Fragoulis A, Rosen C, et al. Interplay between vascular endothelial growth factor (VEGF) and nuclear factor erythroid 2-related factor-2 (Nrf2): implications for preeclampsia. J Biol Chem 2011; 286: 42863-72.

49. Chigusa Y, Tatsumi K, Kondoh E, et al. Decreased lectin-like oxidized LDL receptor 1 (LOX-1) and low Nrf2 activation in placenta are involved in preeclampsia. J Clin Endocrinol Metab 2012; 97: E1862-70.

50. Tong S, Kaitu'U-Lino TJ, Onda K, et al. Heme oxygenase- 1 is not decreased in preeclamptic placenta and does not negatively regulate placental soluble fms-like tyrosine kinase-1 or soluble endoglin secretion. Hypertension 2015; 66: 1073-81. 